\title{
Online Model Regression for Nonlinear Time-Varying Manufacturing Systems
}

\author{
Jinwen $\mathrm{Hu}^{\mathrm{a}}$, Min Zhou ${ }^{\mathrm{b}}$, Xiang $\mathrm{Li}^{\mathrm{c}}$, Zhao $\mathrm{Xu}^{\mathrm{a}}$ \\ ${ }^{a}$ Northwestern Polytechnical University, Xi'an, China \\ ${ }^{\mathrm{b}}$ National University of Singapore, Singapore \\ ${ }^{\mathrm{c}}$ Singapore Institute of Manufacturing Technology, Singapore
}

\begin{abstract}
This paper addresses the online modelling for time-varying manufacturing systems with random unknown model variations between production batches. By modeling the system as a Gaussian process, we first apply the standard Gaussian process regression (GPR) method for estimating the system model, which provides the optimal model estimate with the minimum mean square error (MSE). Then, an iterative form of the method is derived which is more computation efficient but maintains the estimation optimality. However, such optimality is obtained by continuously updating the covariances between the estimated model values and the measurements, which would make the storage and computation unaffordable when the control input can vary within an infinite control space. Due to such a limitation, a suboptimal interactive GPR method is further proposed by trading off the computation efficiency and the estimation accuracy, where the trade-off can be tuned by a designed parameter. Finally, effectiveness and performance of the proposed methods are demonstrated via both simulation and case study by comparing to the conventional nonlinear modelling methods.
\end{abstract}

Key words: Model regression; Gaussian process regression; manufacturing systems.

\section{Introduction}

In the manufacturing industry, model regression is always a tough issue which is come across by the manufacturers when they want to optimize the production performance, improve the product quality or do failure prognosis for the machines [4]. Nowadays, the manufacturing system are getting more and more complex due to the highly automated and integrated components. As a result, the physical models are hard to derive or even intractable [22]. The method of design of experiments (DOE) or empirical knowledge can help build an initial reference system model for common usage. However, the machine conditions most often are changing from time to time in different environment or with different task allocation [36]. The machines may also suffer from sudden

\footnotetext{
* This paper was partially presented at the 13th International Conference on Control, Automation, Robotics and Vision (ICARVC2014). Corresponding author J. Hu.

Email addresses: hujinwen@nwpu.edu. cn (Jinwen Hu), zhoum@u.nus.edu (Min Zhou), xli@simtech.a-star.edu.sg (Xiang Li), zhaoxu@nwpu.edu.cn (Zhao Xu).
}

Preprint submitted to Automatica shifts due to component failure and continuous gradual drifts due to normal wear [35] in the daily batch productions. Therefore, manufacturers need an online model regression method to track the model variations of the systems so that they can always keep a correct view from input to output and thus better control their product quality.

Online model regression methods (also known as online learning or adaptive modelling methods) have been studied for many years. Some are focusing on the model that can reveal the overall system behavior including its transient and steady state performance, while the others are only focusing on the model of the steady state $[3,21,2,47,45]$. In general, the former is more difficult as it requires more information to build a transient response model which usually involves integration and differentiation. However, in many manufacturing processes, especially for the batch processes, knowing the steady state model is enough since they use fixed set points as inputs of the machines during the production of each batch, which is known as supervisory control. In such a case, the steady state input-output correlation can be built easily by using many current existing data mining meth-

27 November 2016 
ods such as the partial least squares [23,18], neural networks (NN) [34,33,6], fuzzy regression $[17,25,39]$, curve fitting $[44,24,15,16]$, local regression $[8,9]$, Gaussian process regression (GPR) $[43,42,38,41,49,46]$, etc. In many batch manufacturing processes such as vapor phase epitaxy, lithography, and chemical mechanical planarization in the semiconductor industry, the set points (or so-called recipes) are tuned between batches to improve output quality against unknown system shift and drift $[35,11,5,13]$. The parameters of such time-varying system thus need to be estimated based on the batch production data.

So far in the literatures, most online model regression methods are focused on fast computation when data are coming in real-time, rather than on the adaptation of the methods to model variations. For example, the online $\mathrm{NN}$ or kernel-based learning methods in $[48,26,28,19,12,31,32]$, and the online GPR methods in $[27,43,37,42,7,30]$ provide iterative calculations for sequential data processing so as to reduce the computation load. Specifically in [7], a selective recursive Gaussian process modelling algorithm is proposed, which adaptively selects the data of a given size that are most efficient in reducing the estimation uncertainty so that the computation load is constrained when online streaming data are coming in. In most current works on batch-to-batch control, the conventional model regression methods are only used for obtaining an initial estimate of the system model using historical data during which the model variations are ignored $[20,35,29]$. The estimated model is then used as a benchmark to identify the model variation of each batch, where the models are usually assumed to be linear. However, such a way of regression modelling is not accurate and reliable since the data of historical batches also suffer from model variations which can not be ignored. Therefore, it is required to design a new online model regression method which takes into account such variations in all batches of production. The key challenge in dealing with the model regression of a time-varying nonstationary process is how to adaptively fuse the data with time-varying nonlinear spatio-temporal correlations.

In this paper, we mainly study the online model regression for time-varying systems under the framework of GPR [43]. This topic is studied due to the following reasons. First, compared with other model regression methods, the GPR method is more general since it is applicable for not only deterministic systems but also stochastic systems and it tells not only what the estimated model is, but also how good the estimation is by its associated variance. Second, unlike other nonlinear model regression methods which only aim to find a good match between the inputs and the outputs, the GPR method explains in-depth data correlations and can be easily combined with Bayesian inference to do forecasting [40]. Third, the standard GPR method is not applicable for online regression with an infinite number of set points in that the dimension of data matrix will go up to infinity if the real-time production data stream in. Finally, the existing modifications of GPR method are mainly designed for reducing computation load and have not considered the effect of model variations.

The main contribution of this paper is that an online model regression method is designed for nonlinear timevarying systems, which is computation efficient in estimating the output values and also provides a confidence evaluation on the model estimates. It can be applied in the modeling of complex nonlinear manufacturing processes with unknown random shifts and drifts, where the model estimate is continuously updated using online input and output data. First, we directly extend the standard GPR method for model regression of time-varying systems with batch data processing, which gives the optimal estimate with minimum mean square error (MSE). Second, the extended method with batch data processing is further modified into an iterative form so that it is more computation efficient for online regression. However, in order to keep the estimation optimality, the covariance between any two model outputs must be continuously updated along with the model estimate, which requires a large storage space. Third, regarding such a limitation, a new suboptimal iterative GPR method is proposed so that both computation and storage requirements are affordable at each iteration. In the meantime, the asymptotic estimation stability is guaranteed when model variation exists. Finally, the proposed methods are validated via simulation and case study as compared with other conventional model regression methods.

The paper is organized as follows. In Section 2, basic assumptions are given and problem is formulated. The method development is illustrated in Section 3. In Section 4, simulation and case study are implemented to demonstrate the effectiveness of the proposed methods. Section 5 draws the conclusions.

\section{Basic Assumptions and Problem Formulation}

In this paper, we consider the nonlinear system model $f_{t}\left(u_{t}\right): \mathbb{R}^{q} \mapsto \mathbb{R}$, where $t$ is the discrete time index number corresponding to the time at which the quantities are evaluated, $u_{t}$ is the control input vector selected from a predefined control space $\mathbb{U} \subset \mathbb{R}^{q}$ and $f_{t}$ is some concerned quality characteristic. There can be more than one quality characteristics, but each of which is individually considered. Thus, without loss of generality $f_{t}$ is assumed to be a scalar. At each evaluation time $t$ during the manufacturing process, the quality characteristic is measured and the following measurement is obtained:

$$
z_{t}=f_{t}\left(u_{t}\right)+v_{t}
$$

where $v_{t}$ is the measurement noise and its variance is denoted by $\sigma_{v}^{2}$. 
Note that in the manufacturing applications, $t$ may represent the batch or run number of production so that $z_{t}$ represents the sample mean of all the output/quality measurements taken for the $t$-th batch [20]. In this paper, we do not consider the change of process setting or measurements within a production batch. The function $f_{t}$ denotes the unknown nonlinear system model which is time-varying. The control settings and quality measurements of the process are usually stored in a database. To use such information, denote by $U_{t}$ a matrix enclosing all the control inputs up to time $t$ with the $i$-th column being $u_{i}(i=1,2, \ldots, t)$, and similarly denote by $Z_{t}$ a vector with the $i$-th entry being $z_{i}$.

In the real manufacturing processes, engineers can always get some prior model estimate by design of experiments or domain physical knowledge during the initial stage of production. Suppose the initial estimated system model is given by

$$
\hat{f}_{0}(u)=\mathbf{m}(u)
$$

where $\hat{f}_{t}(u)$ denotes the estimate of $f_{t}(u)$ at time $t$. Further, define $\tilde{f}_{t}(u)=f(u)-\hat{f}_{t}(u)$ as the estimation error and $\mathrm{E}\left[\left\|\tilde{f}_{t}(u)\right\|^{2}\right]$ as the mean square error of $\hat{f}_{t}(u)$ throughout the paper, where $\|\bullet\|$ denotes the Euclidean norm. In reality, $\tilde{f}_{0}(u)$ may include some unmodelled insignificant terms such as the high order effects in the design of experiments and some estimation bias due to the lack of knowledge about the entire system. Since they are all unknown, $\tilde{f}_{0}(u)$ can be assumed as a zero-mean Gaussian random variable, i.e., $\tilde{f}_{0}(u) \sim \mathcal{N}\left(0, \sigma_{f}^{2}\right)$. Therefore, the initial model of the system is a random function subject to

$$
f_{0}(u) \sim \mathcal{N}\left(\mathbf{m}(u), \sigma_{f}^{2}\right)
$$

A common phenomenon in the manufacturing process is the model shift and drift due to, e.g., sudden disturbances and gradual machine degradation which are unknown and random. Such phenomenon can be modeled as a random walking:

$$
f_{t}(u)=f_{t-1}(u)+\omega_{t-1}
$$

where $\omega_{t}$ is an unknown random variable which includes the unknown random model shift and drift. The variance of $\omega_{t}$ is denoted by $\sigma_{\omega}^{2}$. By this model, it is assumed that the profile of $f_{t}(u)$ does not change as conventionally assumed in batch-to-batch control [35]. $v_{t}$ and $\omega_{t}$ are both assumed to white Gaussian processes with zero means and subject to

$$
\mathrm{E}\left[\begin{array}{l}
v_{t} \\
\omega_{t}
\end{array}\right]\left[\begin{array}{c}
v_{l} \\
\omega_{l}
\end{array}\right]^{\mathrm{T}}=\left[\begin{array}{cc}
\sigma_{v}^{2} \delta_{t l} & 0 \\
0 & \sigma_{\omega}^{2} \delta_{t l}
\end{array}\right]
$$

where $\delta_{t l}$ is the Kronecker delta and "T" denotes the transpose operation. $v_{t}$ and $\omega_{t}$ are both uncorrelated to $f_{0}(u)$. Note that such assumptions are only made for the optimality of the proposed method, which will not impede the proposed method to be applied under other conditions. An example that $\omega_{t}$ is not zero-mean white Gaussian process is shown in Section 4.

Since the process $f_{t}(u)$ may vary with the control input $u$ and time $t$, it is actually a spatio-temporal process. We borrow the idea of spatial-temporal modelling of the stochastic field which is widely used in geostatistics to deal with the model regression of $f_{t}(u)$, such as the simple kriging, where the covariance function needs to be calculated first. There are usually two ways to calculate the covariance of the process, empirical evaluation based on the data or presuming the form of variance function [43]. The latter approach can provide smooth variance function and works faster when the data volume is huge, which therefore is adopted in this paper. One of the widely used forms of covariance function is the Gaussian function defined as follows:

$$
\begin{aligned}
\operatorname{cov}\left(f_{0}(\boldsymbol{a}), f_{0}(\boldsymbol{b})\right) & =\sigma_{f}^{2} \exp \left(-\frac{\|\boldsymbol{a}-\boldsymbol{b}\|^{2}}{h^{2}}\right) \\
& \triangleq \sigma_{f}^{2} K(\boldsymbol{a}, \boldsymbol{b}),
\end{aligned}
$$

where $\boldsymbol{a}, \boldsymbol{b} \in \mathbb{U}$. Model (5) implies that the larger the values of $\|\boldsymbol{a}-\boldsymbol{b}\|$, the less the correlation between $f_{0}(a)$ and $f_{0}(b)$. For concise notations in this paper, it is defined for two random vectors $\mathbf{w} \in \mathbb{R}^{m}$ and $\mathbf{v} \in \mathbb{R}^{n}$ that $\operatorname{cov}(\mathbf{w}, \mathbf{v})$ is a $m \times n$ matrix whose $i j$-th entry is $\operatorname{cov}\left(\mathbf{w}_{i}, \mathbf{v}_{j}\right)$ where $\mathbf{w}_{i}$ and $\mathbf{v}_{j}$ are respectively the $i$-th entry of $\mathbf{w}$ and the $j$-th entry of $\mathbf{v}$.

Under the above assumptions, we are going to develop an online model regression method so that for any given input $u$, its corresponding output $f_{t}(u)$ can be estimated based on the real-time measurements of the time-varying system defined by (4).

\section{$3 \quad$ Methodology}

The conventional Gaussian process regression (GPR) (or so-called kriging method in some geostatistical applications) makes use of all the currently available data to calculate the optimal estimate, i.e., the estimate with the minimum MSE. Moreover, it is often assumed that the process model $f(u)$ is time-invariant. In this paper, we will generalize the GPR method into time-varying systems defined by (4) and more importantly, develop a computationally affordable method for online regression when the total data volume can increase to infinity. 


\subsection{GPR for Time-Varying Systems}

In this section, we generalize the GPR method in the regression modeling of time-varying systems defined by (4), which gives the optimal model estimate by making use of all current available data. The result is described by the following lemma.

Lemma 1 The estimate of $f_{t}(u)(u \in \mathbb{U})$ based on $Z_{t}$ $(t>0)$ with the minimum MSE is given by

$$
\hat{f}_{t}(u)=\mathbf{m}(u)+\xi_{t}^{\mathrm{T}} \Sigma_{t}^{-1}\left(Z_{t}-\mathbf{m}\left(U_{t}\right)\right)
$$

and its MSE is given by

$$
\mathrm{E}\left[\left\|\tilde{f}_{t}(u)\right\|^{2}\right]=\sigma_{f}^{2}+\sigma_{\omega}^{2} t-\xi_{t}^{\mathrm{T}} \Sigma_{t}^{-1} \xi_{t},
$$

where

$$
\begin{aligned}
\xi_{t} & =\sigma_{f}^{2} K\left(U_{t}, u\right)+\sigma_{\omega}^{2} \boldsymbol{n}_{t}, \\
\Sigma_{t} & =\sigma_{f}^{2} K\left(U_{t}, U_{t}\right)+\sigma_{\omega}^{2} \boldsymbol{N}_{t}+\sigma_{v}^{2} I,
\end{aligned}
$$

and $\mathbf{m}\left(U_{t}\right)=\left[\mathbf{m}\left(u_{1}\right), \mathbf{m}\left(u_{2}\right), \ldots, \mathbf{m}\left(u_{t}\right)\right]^{\mathrm{T}}, \boldsymbol{n}_{t}=$ $[1,2, \ldots, t]^{\mathrm{T}}, \boldsymbol{N}_{t}$ is a $t \times t$ matrix with the $i j$-th entry equal to $\min (i, j), I$ is the identity matrix of appropriate dimension, $K\left(u, U_{t}\right)=\left(K\left(U_{t}, u\right)\right)^{\mathrm{T}}$ is a $1 \times t$ vector with the $i$-th entry being $K\left(u, u_{i}\right)$ and $K\left(U_{t}, U_{t}\right)$ is a matrix with the $i$-th row equal to $K\left(u_{i}, U_{t}\right)$.

PROOF. See Appendix A.

Lemma 1 gives the optimal estimate based on all the current available data. It makes the analysis easy since it directly gives the explicit form in terms of the initial condition and all the historical data. However, it is not applicable for online model regression where the data volume can reach infinity. A suitable solution is to design an iterative regression scheme which computes a model estimate by incorporating only the latest measurements in a short time interval. In the next section, we will further look into the design of an iterative regression scheme.

\subsection{Online Iterative Regression}

In [10], an iterative GPR method was proposed, but only for time-invariant systems. In this section, we aim to design an iterative regression scheme that provides the same optimal estimate as in (6) for the time-varying system defined by (4). The iteration of the model estimate is given in the following theorem and the regression scheme is shown in Alg. 1.
Theorem 1 The estimate of $f_{t}(u)(u \in \mathbb{U})$ based on $Z_{t}(t>0)$ with the minimum MSE can be calculated iteratively as follows:

$$
\begin{aligned}
& \hat{f}_{t+1}(u)=\hat{f}_{t}(u)+ \\
& \quad \frac{R_{t}\left(u, u_{t+1}\right)+\sigma_{\omega}^{2}}{R_{t}\left(u_{t+1}, u_{t+1}\right)+\sigma_{\omega}^{2}+\sigma_{v}^{2}}\left(z_{t+1}-\hat{f}_{t}\left(u_{t+1}\right)\right),
\end{aligned}
$$

where $R_{t}(\boldsymbol{a}, \boldsymbol{b})(\boldsymbol{a}, \boldsymbol{b} \in \mathbb{U})$ is iteratively computed by

$$
\begin{aligned}
& R_{t+1}(\boldsymbol{a}, \boldsymbol{b})=R_{t}(\boldsymbol{a}, \boldsymbol{b})+\sigma_{\omega}^{2}- \\
& \frac{\left(R_{t}\left(\boldsymbol{a}, u_{t+1}\right)+\sigma_{\omega}^{2}\right)\left(R_{t}\left(\boldsymbol{b}, u_{t+1}\right)+\sigma_{\omega}^{2}\right)}{R_{t}\left(u_{t+1}, u_{t+1}\right)+\sigma_{\omega}^{2}+\sigma_{v}^{2}} .
\end{aligned}
$$

with the initial conditions that $\hat{f}_{0}(u)=\mathbf{m}(u)$ and $R_{0}(\boldsymbol{a}, \boldsymbol{b})=\sigma_{f}^{2} \exp \left(-\frac{\|\boldsymbol{a}-\boldsymbol{b}\|^{2}}{h^{2}}\right)$. The MSE of $\hat{f}_{t}(u)$ is given by

$$
\mathrm{E}\left[\left\|\tilde{f}_{t}(u)\right\|^{2}\right]=R_{t}\left(u_{t}, u_{t}\right) .
$$

PROOF. See Appendix B.

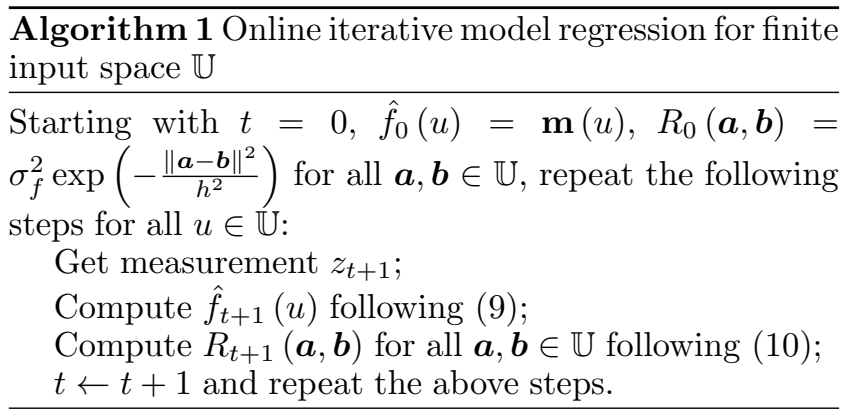

Alg. 1 shows the skeleton of the online iterative model regression scheme which can process the data in sequential order. Though it is mainly designed for the time-varying systems with real-time measurements, it can also be applied in the time-invariant systems with big volume of data where the computation is time exhausting. However, it still suffers from some limitations. If the control input space $\mathbb{U}$ is infinite from which the sampling input $u_{t}(t>0)$ is drawn, then it is only required to maintain in the memory the latest values of $\hat{f}_{t}(u)$ and $R_{t}(\boldsymbol{a}, \boldsymbol{b})$ for all $u, \boldsymbol{a}, \boldsymbol{b} \in \mathbb{U}$ at each iteration, and update their values by (9) and (10). Although in many real manufacturing processes such as in the bio-chemical manufacturing processes, $\mathbb{U}$ is usually finite where each setting of the machine input parameters is normally fixed for a certain type of product, there might be cases in which the machine input parameters can be tuned arbitrarily within a continuous range. In such cases, $\mathbb{U}$ is infinite and the regression scheme is difficult to apply since a computer with limited storage space cannot memorize 
an infinite number of values of $\hat{f}_{t}(u)$ and $R_{t}(\boldsymbol{a}, \boldsymbol{b})$ for all $u, \boldsymbol{a}, \boldsymbol{b} \in \mathbb{U}$. Even if $\mathbb{U}$ is finite but with a high cardinality, the computation and storage requirement is still challenging. Therefore, it is necessary to design a new model regression scheme that can run with limited computation and storage resources, which will be discussed in the next section.

\subsection{Regression with Computation Limitations}

As illustrated in the above section, the iterative regression scheme described in Theorem 1 cannot be directly applied due to the difficulty in updating the values of $\hat{f}_{t}$ and $R_{t}$ when $\mathbb{U}$ is infinite. A reasonable way of dealing with such a problem is to make a trade-off between the estimation performance and the computation cost. That is to let computer store and update the estimates of finite number of model values, and any other model values can be estimated (only when required) using the former stored estimates. In the meantime, the covariance information between any two model values will not be stored and updated due to the infinite or high cardinality of $\mathbb{U}$.

The general procedure of such scheme is stated as follows: 1) Select a finite set $\mathbb{U}_{F} \subset \mathbb{U}$ over which the model function $f_{t}(u)$ is to be estimated based on the measurements, and keep these estimates in storage; 2) Compute the estimates of $f_{t}(u)$ for $u \in \mathbb{U} \backslash \mathbb{U}_{\mathrm{F}}$ (only when required) based on the obtained estimates over $\mathbb{U}_{\mathrm{F}}$, and don't keep them in storage; 3) When new measurement comes, only update the estimates of $f_{t}(u)$ for $u \in \mathbb{U}_{\mathrm{F}}$ which are stored in the memory.

Following this scheme, it can be foreseen that the model estimate is no more the optimal estimate since the estimates of $f_{t}(u)$ for $u \in \mathbb{U} \backslash \mathbb{U}_{\mathrm{F}}$ are indirectly computed from the measurements and there might be information loss in such an indirect fusion of measurements. Next, we will give rigorous mathematical formulations of the three steps, and the main calculations are summarized in Alg. 2.

Define $Z_{L, t}(t \geqslant 1)$ to be a $\tau \times 1$ vector where $L$ is a user defined positive integer and $\tau=\min (L, t)$. The $i$ th entry of $Z_{L, t}$ is $z_{t-\tau+i}$. Specifically, $Z_{1, t}=z_{t}$ when $L=1$. Hence, $L$ stands for the time length of data memory. In the same way, we can define $U_{L, t}, \mathbf{m}\left(U_{L, t}\right)$, $\boldsymbol{n}_{L, t}$ and $\boldsymbol{N}_{L, t}$ following the definitions of $U_{t}, \mathbf{m}\left(U_{t}\right), \boldsymbol{n}_{t}$ and $\boldsymbol{N}_{t}$ in (8), i.e., to cut them into vectors or matrices with appropriate dimensions. Denote the cardinality of $\mathbb{U}_{\mathrm{F}}$ by $\lambda$ and its elements by $\bar{u}_{i}(i=1,2, \ldots, \lambda)$. Then, we can define $U_{\mathrm{F}}=\left[\bar{u}_{1}, \bar{u}_{2}, \ldots, \bar{u}_{\lambda}\right]^{\mathrm{T}}, f_{t}\left(U_{\mathrm{F}}\right)=$ $\left[f_{t}\left(\bar{u}_{1}\right), f_{t}\left(\bar{u}_{2}\right), \ldots, f_{t}\left(\bar{u}_{\lambda}\right)\right]^{\mathrm{T}}$ and similarly for $\hat{f}_{t}\left(U_{\mathrm{F}}\right)$, $\tilde{f}_{t}\left(U_{\mathrm{F}}\right)$ and $\mathbf{m}\left(U_{\mathrm{F}}\right)$ all of which are $\lambda \times 1$ vectors.

Given $Z_{L, t+1}$ at time $t+1(t \geqslant 0)$, we can do the following estimation based on measurements similar to (6):
1) Measurement update for $u \in \mathbb{U}_{F}$ and $t \geqslant 0$ :

$$
\begin{aligned}
& \phi_{t+1}=\mathrm{M}\left(U_{\mathrm{F}}\right)+\zeta_{t+1}^{\mathrm{T}} \Xi_{t+1}^{-1}\left(Z_{L, t+1}-\mathbf{m}\left(U_{L, t+1}\right)\right), \\
& \Gamma_{t+1}=\sigma_{f}^{2} K\left(U_{\mathrm{F}}, U_{\mathrm{F}}\right)+\sigma_{\omega}^{2}(t+1) \mathbf{1 1}^{\mathrm{T}}-\zeta_{t+1}^{\mathrm{T}} \Xi_{t+1}^{-1} \zeta_{t+1},
\end{aligned}
$$

where

$$
\begin{aligned}
\zeta_{t+1} & =\sigma_{f}^{2} K\left(U_{L, t+1}, U_{\mathrm{F}}\right)+\sigma_{\omega}^{2} \boldsymbol{n}_{L, t+1} \mathbf{1}^{\mathrm{T}}, \\
\Xi_{t+1} & =\sigma_{f}^{2} K\left(U_{L, t+1}, U_{L, t+1}\right)+\sigma_{\omega}^{2} \boldsymbol{N}_{L, t+1}+\sigma_{v}^{2} I
\end{aligned}
$$

and $\mathbf{1}$ is a column vector of appropriate dimension with all entries equal to one. It is easy to see that $\phi_{t+1}=$ $\mathrm{E}\left[f_{t+1}\left(U_{\mathrm{F}}\right) \mid Z_{L, t+1}\right]$ and $\Gamma_{t+1}$ is the MSE of $\phi_{t+1}$.

The measurement update step 1) has not taken into account $\hat{f}_{t}\left(U_{\mathrm{F}}\right)$, the estimate obtained at the time $t$ compared with (9). To further combine $\hat{f}_{t}\left(U_{\mathrm{F}}\right)$ and $\phi_{t+1}$ into one estimate as a fusion of the two sources of information, the covariance intersection (CI) method can be applied which was initially proposed to seek the optimal fusion of two estimates with unknown correlation $[1,14]$.

To apply the CI method, define an auxiliary variable $G_{t}\left(U_{\mathrm{F}}\right)$ which can be seen as an estimate of the MSE of $\hat{f}_{t}\left(U_{\mathrm{F}}\right)$. Given $\hat{f}_{t}\left(U_{\mathrm{F}}\right), G_{t}\left(U_{\mathrm{F}}\right), \phi_{t+1}$ and $\Gamma_{t+1}(t \geqslant$ $L)$ with initial conditions that $\hat{f}_{0}\left(U_{\mathrm{F}}\right)=\mathbf{m}\left(U_{\mathrm{F}}\right)$ and $G_{0}\left(U_{\mathrm{F}}\right)=\sigma_{f}^{2} K\left(U_{\mathrm{F}}, U_{\mathrm{F}}\right)$, we can get the following estimate update:

2) Estimate update for $u \in \mathbb{U}_{F}$ :

$$
\begin{aligned}
\hat{f}_{t+1}\left(U_{\mathrm{F}}\right) & = \begin{cases}G_{t+1}\left(U_{\mathrm{F}}\right)\left(\Lambda_{1} \hat{f}_{t}\left(U_{\mathrm{F}}\right)+\Lambda_{2} \phi_{t+1}\right), & \text { if } t \geqslant L ; \\
\phi_{t+1}, & \text { otherwise, }\end{cases} \\
G_{t+1}\left(U_{\mathrm{F}}\right) & = \begin{cases}\left(\Lambda_{1}+\Lambda_{2}\right)^{-1}, & \text { if } t \geqslant L ; \\
\Gamma_{t+1}, & \text { otherwise, }\end{cases}
\end{aligned}
$$

where

$$
\begin{aligned}
& \Lambda_{1}=\alpha_{t+1}\left(G_{t}\left(U_{\mathrm{F}}\right)+\sigma_{\omega}^{2} \mathbf{1 1}^{\mathrm{T}}\right)^{-1} \\
& \Lambda_{2}=\left(1-\alpha_{t+1}\right) \Gamma_{t+1}^{-1}
\end{aligned}
$$

where $0 \leqslant \alpha_{t+1} \leqslant 1$ is a fusion factor to be designed. In (14), we directly let $\hat{f}_{t+1}(u)=\phi_{t+1}$ for $0 \leqslant t<$ $L$, since in this case $\phi_{t+1}$ has already been the optimal estimate with the minimum MSE according to (6). By implementing the measurement update (12) and (14), we can get the following conclusions.

Lemma 2 The MSE of $\hat{f}_{t}\left(U_{\mathrm{F}}\right)$ is uniformly bounded by $\operatorname{Tr}\left(G_{t}\left(U_{F}\right)\right)$, where $\operatorname{Tr}(\bullet)$ denotes the trace of the en- 
closed matrix.

PROOF. It has been proven in [1] that $G_{t}\left(U_{\mathrm{F}}\right)-$ $\mathrm{E}\left[\tilde{f}_{t}\left(U_{\mathrm{F}}\right)\left(\tilde{f}_{t}\left(U_{\mathrm{F}}\right)\right)^{\mathrm{T}}\right]$ is always nonnegative definite, which is directly followed by the conclusion.

Theorem 2 There exists a solution of the time series $\left\{\alpha_{t}: 0 \leqslant \alpha_{t} \leqslant 1, t>0\right\}$ such that the MSE of $\hat{f}_{t}\left(U_{F}\right)$ and $\operatorname{Tr}\left(G_{t}\left(U_{F}\right)\right)$ are uniformly bounded for all $t>0$.

PROOF. See Appendix C.

Remark 1 Lemma 2 shows that $\operatorname{Tr}\left(G_{t}\left(U_{F}\right)\right)$ can be used as a conservative estimate of the true MSE of $\hat{f}_{t}\left(U_{F}\right)$. Theorem 2 further shows that by implementing the proposed estimation scheme $G_{t}\left(U_{F}\right)$ and the MSE of $\hat{f}_{t}\left(U_{F}\right)$ do not diverge as the time approaches infinity. In the proof of Theorem 2, only the bound for a special case $\alpha_{t} \equiv 0$ is given. Actually, $\alpha_{t}$ can be tuned to minimize $\operatorname{Tr}\left(G_{t}\left(U_{F}\right)\right)$ so that it is a tight estimate of the MSE of $\hat{f}_{t}\left(U_{F}\right)$. Due to the computation complexity of such optimization, fast computation techniques for calculation of suboptimal $\alpha_{t}$ have been given in [14] where $\alpha_{t}=\frac{\operatorname{Tr}\left(\left(G_{t}\left(U_{\mathrm{F}}\right)+\sigma_{\omega}^{2} \mathbf{1 1}^{\mathrm{T}}\right)^{-1}\right)}{\operatorname{Tr}\left(\left(G_{t}\left(U_{\mathrm{F}}\right)+\sigma_{\omega}^{2} \mathbf{1 1}^{\mathrm{T}}\right)^{-1}\right)+\operatorname{Tr}\left(\Gamma_{t}^{-1}\right)}$. However, this solution may not be able to guarantee the boundness of $\operatorname{Tr}\left(G_{t}\left(U_{F}\right)\right)$. In our method, we find a suitable $\alpha_{t}$ by a line search within $[0,1]$ which gives the minimum $\operatorname{Tr}\left(G_{t}\left(U_{F}\right)\right)$ among all the searched values.

Next, we need to estimate $f_{t+1}(u)$ for $u \in \mathbb{U} \backslash \mathbb{U}_{\mathrm{F}}$ only using $\hat{f}_{t+1}\left(U_{\mathrm{F}}\right)$ that have been obtained by (14), where the measurements $Z_{t+1}$ are ignored. Intuitively from (6), the easiest estimate of $f_{t+1}(u)$ is given by a linear combination of $\hat{f}_{t+1}\left(U_{\mathrm{F}}\right)$ which is described as follows.

3) Estimate for $u \in \mathbb{U} \backslash \mathbb{U}_{F}$ (if required):

$$
\hat{f}_{t+1}(u)=\mathbf{m}(u)+W(u)\left(\hat{f}_{t+1}\left(U_{\mathrm{F}}\right)-\mathbf{m}\left(U_{\mathrm{F}}\right)\right),
$$

where $W(u)$ is the combination weight matrix. Intuitively, the closer $u$ is to $\bar{u}_{i}$ (i.e., the element in $\mathbb{U}_{\mathrm{F}}$ ), the larger weight should be assigned to $\hat{f}_{t+1}\left(\bar{u}_{i}\right)$. Thus, we design the combination weight matrix as follows:

$$
W(u)=\beta(u) K\left(u, U_{\mathrm{F}}\right)\left(K\left(U_{\mathrm{F}}, U_{\mathrm{F}}\right)\right)^{-1},
$$

where $\beta(u)$ is a normalization coefficient such that $W(u) \mathbf{1}=1$, which is introduced to guarantee that the MSE of $\hat{f}_{t}(u)$ is bounded. Another reason to design such a form of $W(u)$ is to make $\hat{f}_{t}(u)$ obtained in (15) smooth for any $u \in U_{\mathrm{F}}$. That is, (15) also holds if we replace $u$ by $U_{\mathrm{F}}$, so that the estimates of $f_{t}(u)$ for all $u \in U_{\mathrm{F}}$ share a unified analytic model.

Theorem 3 The MSE of the estimate $\hat{f}_{t}(u)$ given by (15) is uniformly bounded for all $t>0$ and $u \in \mathbb{U} \backslash \mathbb{U}_{F}$.

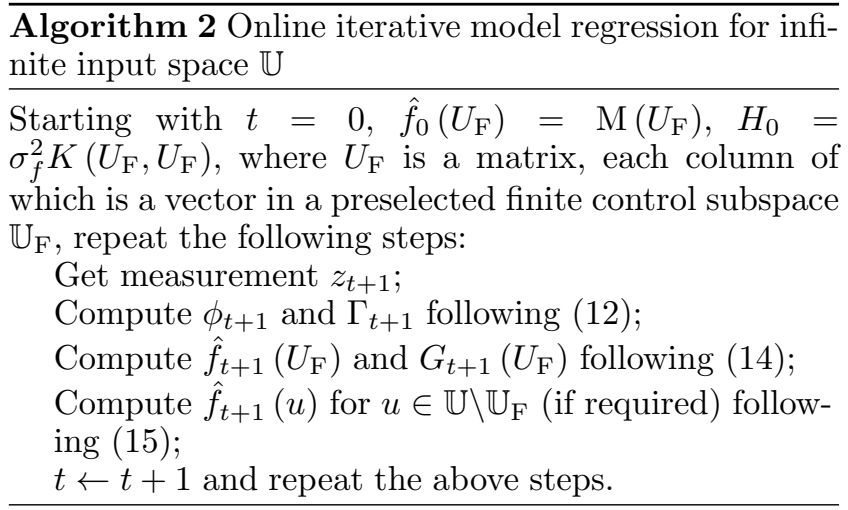

PROOF. See Appendix D.

Remark 2 Compared to Alg. 1, Alg. 2 solves the online model regression for infinite input space $\mathbb{U}$ by introducing a time length $L$ and a finite subspace $U_{F}$. It is obvious to see that if $L=t$ and $U_{F}=\mathbb{U}$, Alg. 2 will give the same optimal estimate as in (6) which is equivalent to that given by Alg. 1. Therefore, with a fixed $L$ and $U_{F} \subset \mathbb{U}$, the estimate is definitely not optimal, but it makes computation and storage affordable for online regression with infinite number of measurements at the cost of estimation performance. In the next section, the algorithm performance will be tested with different time length and with both finite and infinite $\mathbb{U}$.

\section{Simulation and Case Study}

\subsection{Simulation Study}

In this section, we implement simulations to testify the effectiveness of the proposed model regression algorithms. By simulation, the data of a presumed process model can be generated arbitrary number of times, e.g., by Monte Carlo simulations, so that it is easier to testify the stability of algorithms against random model variations. A case study using the data of an actual manufacturing process will be demonstrated in the next section.

Two types of commonly used online modeling algorithms are compared with the proposed algorithms. One is the the online extreme learning machine (ELM) [28] which is a neural network type algorithm and famous for its high computing speed, and the other is the selective recursive Gaussian process modelling algorithm (SRGPMA) proposed in [7] which is based on the GPR method and 
similar to our work. The algorithms are simulated in two different scenarios, i.e., $\mathbb{U}$ is finite and infinite respectively. In Scenario I with finite $\mathbb{U}$, we compare four different algorithms, i.e., the optimal iterative GPR method stated in Alg. 1, the suboptimal iterative GPR method in Alg. 2, the online ELM and SRGPMA. In Scenario II with infinite $\mathbb{U}$, we compare Alg. 2 with the online ELM and SRGPMA, and also evaluate Alg. 2 with different data memory length $L$.

\subsubsection{Simulation Setup}

First, we consider the following process model:

$$
f_{t}\left(x_{1}, x_{2}\right)=\left(x_{1}-10\right)^{2} \sin \left(x_{2}\right)+0.1 x_{1} x_{2}+0.1 t+\varrho_{t},
$$

where the input vector $u=\left[x_{1}, x_{2}\right]^{\mathrm{T}}$. Suppose the sum of the first term in the above equation is known by empirical knowledge and set as the initial estimate, i.e., $\hat{f}_{0}=\mathrm{m}\left(x_{1}, x_{2}\right)=\left(x_{1}-10\right)^{2} \sin \left(x_{2}\right)$. The other terms, $0.1 x_{1} x_{2}, 0.1 t$ and $\varrho_{t} \sim \mathcal{N}(0,1)$, stand for the initial estimation bias, unknown gradual drift and unknown random shift respectively. Note that such a presumed process model does not represent any physical process and is only proposed for easy demonstration of performance evaluation. In actual processes, the shift or drift may not be that large and they are set to be relatively large in this simulation to make the difference more obvious between the algorithms.

In simulation, we set the variances of process and measurement noises respectively as $\sigma_{\omega}^{2}=1, \sigma_{v}^{2}=1$. The variance of initial estimation error is set as $\sigma_{f}^{2}=100$. The width factor $h$ in (1) is set as 2. Then in Scenario I, let $A=\{5,6, \ldots, 15\}, B=\{0,1,2, \ldots, 10\}$ and $\mathbb{U}=A \times B$, where $x_{1} \in A$ and $x_{2} \in B$. Therefore, there are totally 121 points in $\mathbb{U}$. In this scenario, let $\mathbb{U}_{\mathrm{F}}=\mathbb{U}$ and test with two data memory lengths $L=5$ and $L=10$ for Alg. 2. SRGPMA also has a data memory length which is set the same as Alg. 2. In Scenario II, $\mathbb{U}$ is changed to be infinite by redefining $A=[5,15]$, $B=[0,10]$ and letting $\mathbb{U}=A \times B$. For Alg. 2, we keep $\mathbb{U}_{\mathrm{F}}=\{5,6, \ldots, 15\} \times\{0,1,2, \ldots, 10\}$.

In both scenarios, we define the following average MSE over all points and use it as a metric to evaluate the performance of different method:

$$
\text { Error }_{t}=\frac{1}{\mathrm{~N}} \sum_{u \in \mathbb{U}_{\mathrm{F}}} \mathrm{E}\left[\left(\tilde{f}_{t}(u)\right)^{2}\right],
$$

where $\mathrm{N}=121$ is the total number of points in $\mathbb{U}_{\mathrm{F}}$, over which the function values are to be estimated. The expectation in the above equation is averaged over 1000 Monte Carlo simulations, where the input $u_{t}$ is randomly selected from $\mathbb{U}$ at each time instant. In parallel, the average of the computed variances denoted by $\operatorname{Var}_{t}$ is also evaluated:

$$
\operatorname{Var}_{t}=\frac{1}{\mathrm{~N}} \sum_{u \in \mathbb{U}_{\mathrm{F}}} G_{t}(u)
$$

\subsubsection{Simulation Results}

As shown in Fig. 1(a) with finite control space $\mathbb{U}$, Alg. 1 performs the best in terms of the average MSE, and the online ELM performs the worst. Moreover, the average MSEs of online ELM and SRGPMA all diverge. Fig. (b) shows that the average MSEs of Alg. 1 and Alg. 2 are bounded by their corresponding average computed variances, which implies that the computed variance can be taken as a conservative estimate of the actual MSE. Moreover, for Alg. 2, it is shown that the larger the data memory length, the smaller the average MSE and the average computed variance. In fact, Alg. 1 can be seen as the extreme case where $L$ approaches infinity. Fig. 2(a) shows that with infinite control space the average MSEs of online ELM and SRGPMA still diverge, and Fig. 2(b) shows that the average MSE of Alg. 2 is always bounded by its average computed covariance. It is also shown that a larger data memory length leads to a smaller MSE and computed variance for Alg. 2, which may not be true for SRGPMA in the time-varying systems.

The above results verify the conclusions of Theorem 1 and Theorem 3. Moreover, in Alg. 2, users can consider the computed variance as a conservative estimate of the actual MSE. Since the computed variance can be obtained off-line before system setup, one can tune the data memory length off-line to meet the optimal tradeoff between the estimation performance and the computation load.

\subsection{Case Study}

\subsubsection{Industrial Testbed}

It is well known that semiconductor manufacturing industry has a very critical quality control standard and the product quality is sensitive to small process drifts and shifts. Therefore, process engineers need to estimate the process model variation after every batch of production and fine tune the control input parameters for better quality control. In this case study, we apply the proposed method in regression modelling of a chemical vapor deposition (CVD) process in semiconductor manufacturing.

The data of totally 933 batches and 15 recipes were collected from one machine, which include two control input parameters: temperature and deposition time, and one output parameter: layer thickness. Due to the confidentiality issue, the data had been normalized between 


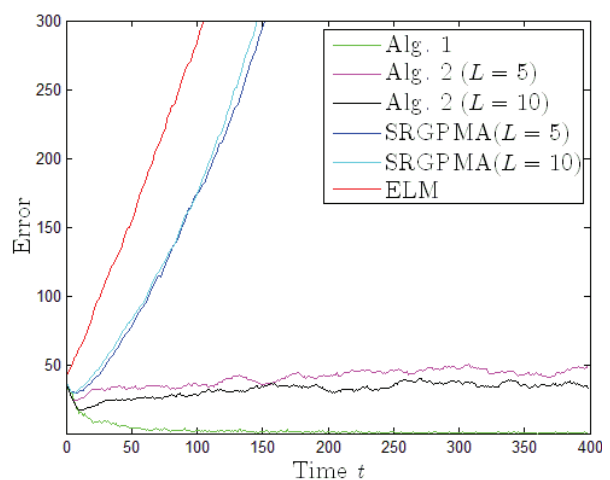

(a) The average MSE.

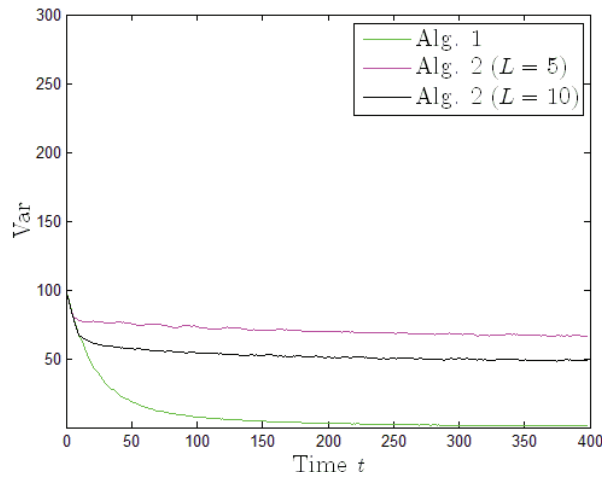

(b) The average computed variance.

Fig. 1. Simulation results of Scenario I with finite $\mathbb{U}$.

-1 and 1 in each dimension before simulation as shown in Fig. 3(a). The measured outputs in batch sequence are shown in Fig. 3(b). The task in this case study is to build the process model so that engineers can use the model to predict the output of the coming batch and fine tune the control input parameters for better quality control.

In this case, the input space $\mathbb{U}$ is infinite because the input parameters can be chosen freely within $[-1,1]$. Thus, we can test and compare the three algorithms as in Scenario II, Section 4.1, i.e., Alg. 2, SRGPMA and ELM. The data memory length is set as $L=5$ for both Alg. 2 and SRGPMA. For Alg. 2, the set $\mathbb{U}_{F}$ can be defined according to the empirical knowledge of engineers. In this case, we can select 15 points into $\mathbb{U}_{F}$, where each point represents an empirical control input setting for a distinctive recipe (as shown in Tab. 1). $\sigma_{\omega}^{2}, \sigma_{v}^{2}$ and $\sigma_{f}^{2}$ are set to be the same as in the simulation part. In the real process, the recipe may change from batch to batch and engineers always tune the inputs in order to make the predicted output meet the target. Therefore, the actual inputs may vary around the points in $\mathbb{U}_{F}$. The initial estimated model is set such that the initial predicted output of each recipe is equal to the target output. Note that the predicted output at $u_{t+1}$ is equal to $\hat{f}_{t}\left(u_{t+1}\right)$. In this case study, we can only obtain the measured outputs $z_{t}$ and have no access to the real model function values $f_{t}\left(u_{t}\right)$. Therefore, the following prediction error

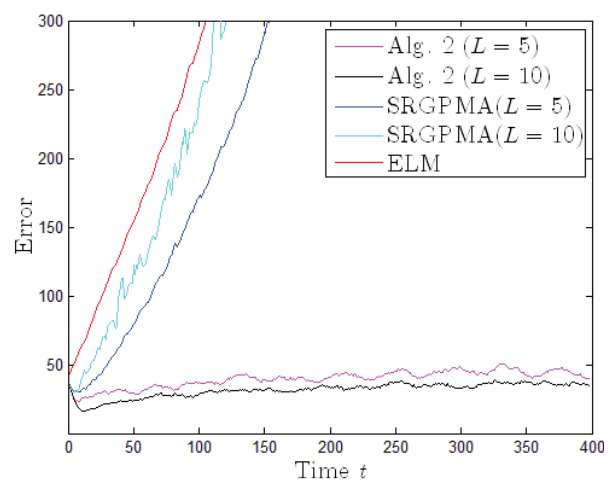

(a) The average MSE.

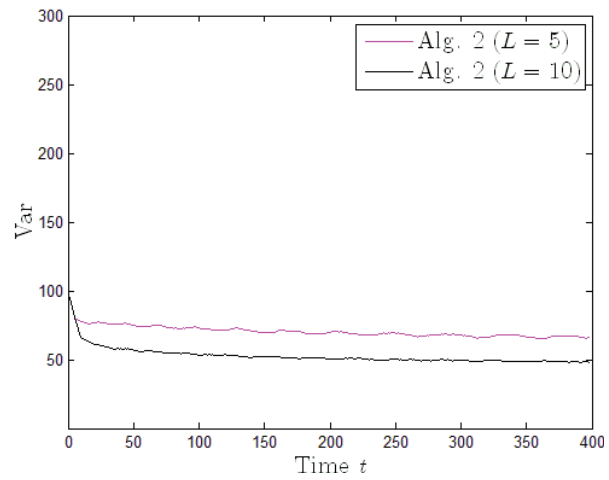

(b) The average computed variance.

Fig. 2. Simulation results of Scenario II with infinite $\mathbb{U}$.

is defined as an index to evaluate the performance of different algorithm:

$$
\operatorname{PreError}_{t}=\left\|\hat{f}_{t-1}\left(u_{t}\right)-z_{t}\right\|^{2} .
$$

Table 1

Empirical input setting and target layer thickness.

\begin{tabular}{|l|l|l|l|l|l|}
\hline Recipe ID & $\# 1$ & $\# 2$ & $\# 3$ & $\# 4$ & $\# 5$ \\
\hline Temperature & -1 & -1 & -1 & -1 & -1 \\
\hline $\begin{array}{l}\text { Deposition } \\
\text { time }\end{array}$ & -0.8826 & -0.7320 & -0.6389 & -0.5244 & -0.3649 \\
\hline $\begin{array}{l}\text { Target layer } \\
\text { thickness }\end{array}$ & -1.0000 & -0.9251 & -0.8717 & -0.8182 & -0.7112 \\
\hline Recipe ID & $\# 6$ & $\# 7$ & $\# 8$ & $\# 9$ & $\# 10$ \\
\hline Temperature & -1 & -1 & 1 & 1 & 1 \\
\hline $\begin{array}{l}\text { Deposition } \\
\text { time }\end{array}$ & 0.9689 & 0.7640 & -0.9944 & -0.7477 & -0.2338 \\
\hline $\begin{array}{l}\text { Target layer } \\
\text { thickness }\end{array}$ & -0.0695 & -0.1765 & -0.7112 & -0.1765 & 1.0000 \\
\hline Recipe ID & $\# 11$ & $\# 12$ & $\# 13$ & $\# 14$ & $\# 15$ \\
\hline Temperature & 1 & 1 & 1 & 1 & 1 \\
\hline $\begin{array}{l}\text { Deposition } \\
\text { time }\end{array}$ & -0.6719 & -0.6148 & -0.4041 & -0.3850 & -0.2993 \\
\hline $\begin{array}{l}\text { Target layer } \\
\text { thickness }\end{array}$ & -0.0160 & 0.1444 & 0.5722 & 0.6791 & 0.7861 \\
\hline
\end{tabular}




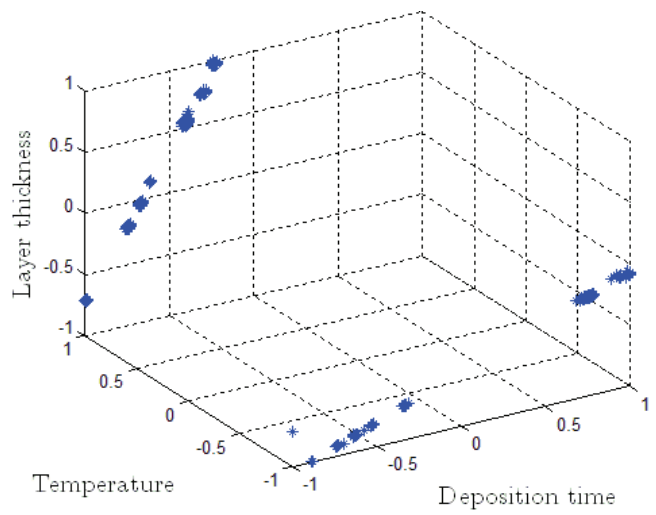

(a) Normalized data points.

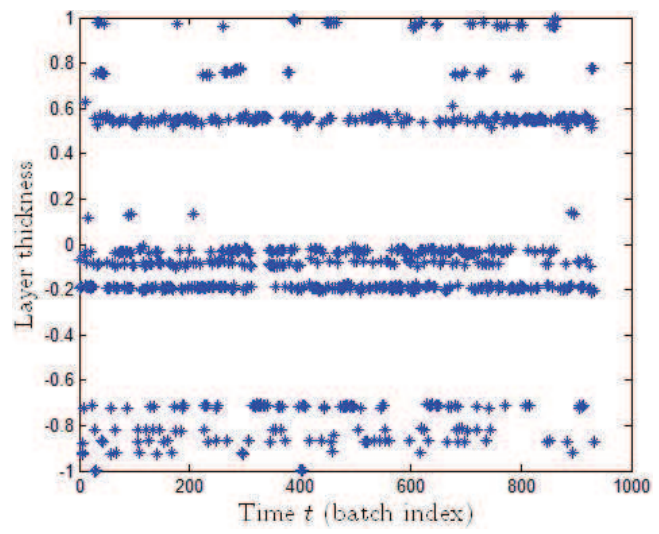

(b) Normalized output in batch sequence.

Fig. 3. Normalized CVD process data.

\subsubsection{Testing Results}

The results of the three algorithms are respectively shown in Fig. 4 to 6 . The average prediction error over the 933 batches, i.e. $\frac{1}{933} \sum_{t=1}^{933}$ PreError $_{t}$, is shown in Tab. 2 and it shows that Alg. 2 performs the best among the three algorithms. Moreover, we also calculate the following percentages to compare the algorithms:

$$
\begin{aligned}
\frac{1}{933} \sum_{t=1}^{933} \mathbb{1}_{\left\{\text {PreError }_{t}^{\mathrm{Alg} 2}<\text { PreError }_{t}^{\mathrm{SRGPMA}}\right\}} & =82.85 \%, \\
\frac{1}{933} \sum_{t=1}^{933} \mathbb{1}_{\left\{\text {PreError }_{t}^{\mathrm{Alg} 2}<\text { PreError }_{t}^{\text {ELM }}\right\}} & =73.42 \%,
\end{aligned}
$$

where $\mathbb{1}_{\{\bullet\}}$ is the indicator function which equals to 1 if the enclosed condition is satisfied and equals to 0 otherwise. The above percentage results illustrate that in $82.85 \%$ of all the batches, the prediction error by Alg. 2 is smaller than that by SRGPMA and in $73.42 \%$ of all the batches smaller than that by ELM, which again verifies the superiority of Alg. 2 over the other two algorithms.

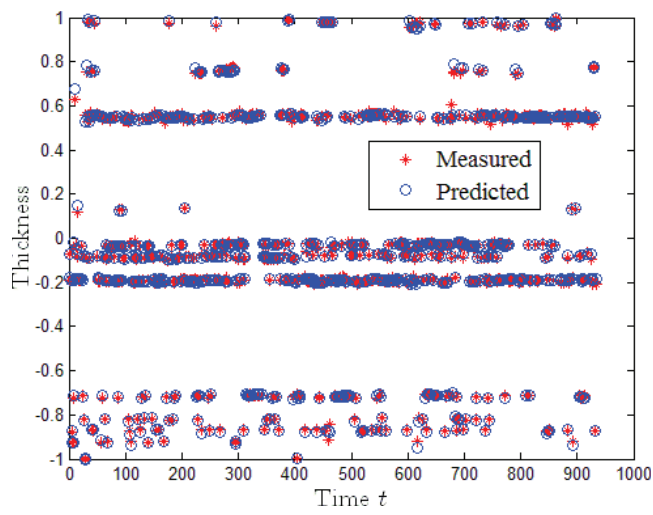

(a) Measured output vs. predicted output.

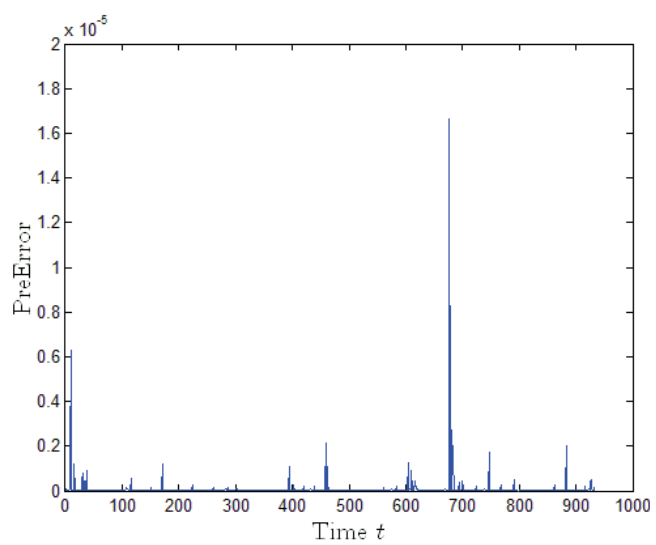

(b) Prediction error.

Fig. 4. Results of Alg. 2.

Table 2

Average prediction error.

\begin{tabular}{|l|l|l|}
\hline Alg. 2 & SRGPMA & ELM \\
\hline $8.6779 \mathrm{e}-05$ & $3.4631 \mathrm{e}-04$ & $4.8147 \mathrm{e}-04$ \\
\hline
\end{tabular}

\section{Conclusions}

In this paper, the standard Gaussian process regression (GPR) method was extended for the model regression of time-varying manufacturing systems with unknown shift and drift. Then, an equivalent iterative form of the extended method was derived so that it is computation affordable when the number of real-time measurements becomes huge. Both the two methods can provide the optimal model estimate. However, maintaining the covariance information between two model outputs that can take an infinite number of values may require infinite storage space. Thus, a suboptimal GPR method was proposed which ignores the correlation between the latest measurement and the historical data as a tradeoff between the storage expense and the estimation optimality. Simulation has been done to verify the effectiveness of the two proposed methods.

In our future work, we will further develop the proposed suboptimal method by considering the effects of condi- 


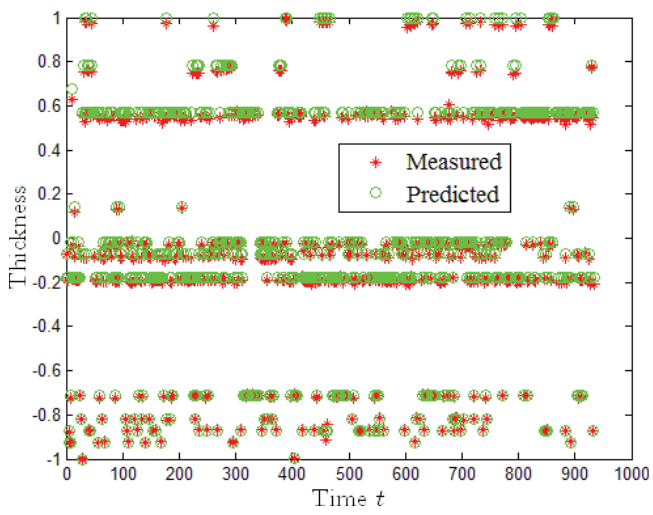

(a) Measured output vs. predicted output.

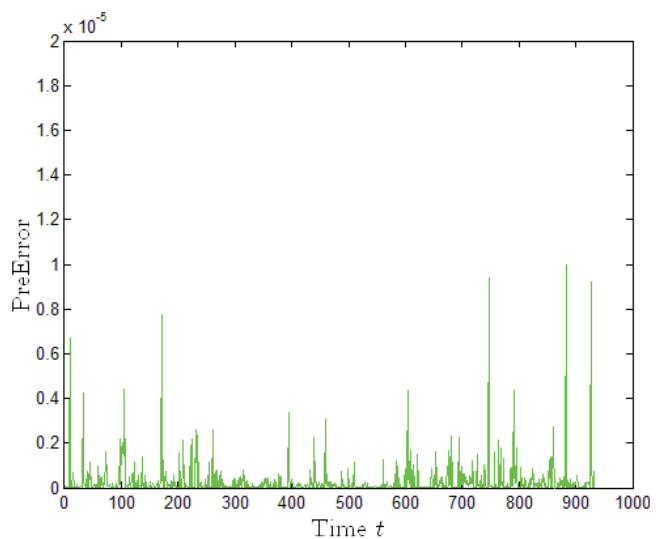

(b) Prediction error.

Fig. 5. Results of SRGPMA.

tion changes on the model regression such as the machine maintenance and breakdown, new type of products, new process design, etc. We will also consider the application of this method in virtual metrology and batch-to-batch control, where the actual physical measurements of the outputs can be replaced by the predicted ones. The potential benefit will be significant reduce of the processing time and cost while the quality control is maintained or even improved by reducing the number of actual physical measurements.

\section{Acknowledgements}

This research is funded by the State Key Laboratory of Geo-information Engineering under grant agreement NO. SKLGIE2015-M-3-4 and is supported by National Science Foundation of China (Grant No. 61473230), National Science Foundation for Young Scholars of China (Grant No. 61603303) and Aviation Science Foundation (Grant No. 2014ZC53030).

\section{References}

[1] P. Arambel, C. Rago, and R. Mehra. Covariance intersection algorithm for distributed spacecraft state estimation. In

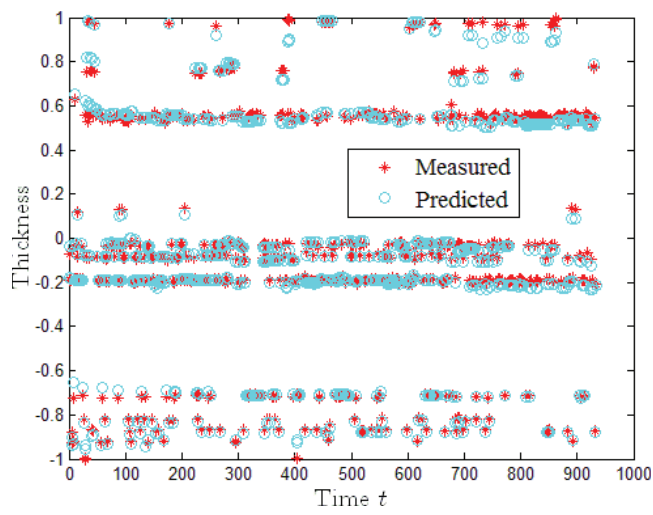

(a) Measured output vs. predicted output.

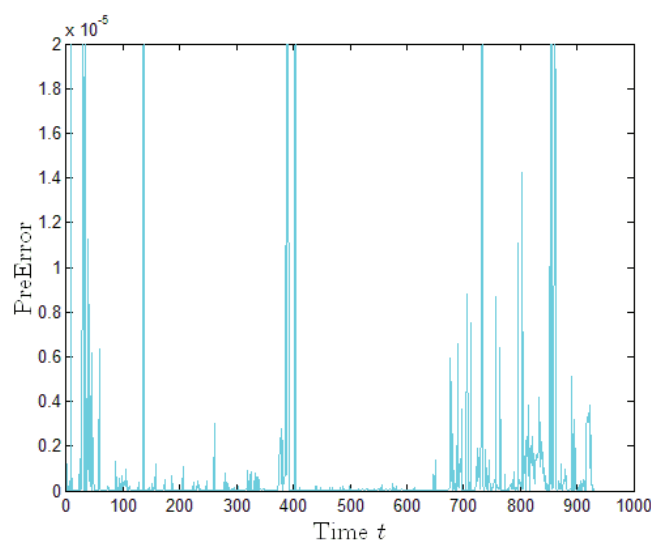

(b) Prediction error.

Fig. 6. Results of ELM.

Proceedings of the American Control Conference, volume 6, pages 4398-4403, 2002.

[2] Karl J Åström and Björn Wittenmark. Adaptive control. Courier Dover Publications, 2013.

[3] Albert Benveniste, Michel Métivier, and Pierre Priouret. Adaptive algorithms and stochastic approximations. Springer Publishing Company, Incorporated, 2012.

[4] John A Buzacott and J George Shanthikumar. Stochastic models of manufacturing systems, volume 4. Prentice Hall Englewood Cliffs, NJ, 1993.

[5] W Jarrett Campbell, Stacy K Firth, Anthony J Toprac, and Thomas F Edgar. A comparison of run-to-run control algorithms. In Proceedings of the American Control Conference, volume 3, pages 2150-2155. IEEE, 2002.

[6] Tianyou Chai, Zhongsheng Hou, Frank L Lewis, Amir Hussain, and Dongbin Zhao. Guest editorial data-based control, modeling, and optimization. IEEE Transactions on Neural Networks, 22(12):2150-2153, 2011.

[7] Lester Lik Teck Chan, Yi Liu, and Junghui Chen. Nonlinear system identification with selective recursive gaussian process models. Industrial \& Engineering Chemistry Research, 52(51):18276-18286, 2013.

[8] William S Cleveland, Susan J Devlin, and Eric Grosse. Regression by local fitting: methods, properties, and computational algorithms. Journal of econometrics, 37(1):87-114, 1988.

[9] William S Cleveland and Clive Loader. Smoothing by local regression: Principles and methods. In Statistical theory and 
computational aspects of smoothing, pages 10-49. Springer, 1996.

[10] Lehel Csató and Manfred Opper. Sparse on-line gaussian processes. Neural computation, 14(3):641-668, 2002.

[11] E Del Castillo. A multivariate self-tuning controller for runto-run process control under shift and trend disturbances. IIE transactions, 28(12):1011-1021, 1996.

[12] Haijin Fan and Qing Song. A sparse kernel algorithm for online time series data prediction. Expert Systems with Applications, 40(6):2174-2181, 2013.

[13] Stacy K Firth, W Jarrett Campbell, Anthony Toprac, and Thomas F Edgar. Just-in-time adaptive disturbance estimation for run-to-run control of semiconductor processes. IEEE Transactions on Semiconductor Manufacturing, 19(3):298-315, 2006.

[14] D. Franken and A. Hupper. Improved fast covariance intersection for distributed data fusion. In Proceedings of the 8th International Conference on Information Fusion, volume 1, page 7, 2005.

[15] Zhun ga Liu, Quan Pan, Jean Dezert, and Arnaud Martin. Adaptive imputation of missing values for incomplete pattern classification. Pattern Recognition, 52:85 - 95, 2016.

[16] Zhun ga Liu, Quan Pan, Jean Dezert, and Grgoire Mercier. Credal c-means clustering method based on belief functions. Knowledge-Based Systems, 74:119 - 132, 2015.

[17] Amir Hossein Gandomi and Amir Hossein Alavi. Multi-stage genetic programming: a new strategy to nonlinear system modeling. Information Sciences, 181(23):5227-5239, 2011.

[18] Paul Geladi and Bruce R Kowalski. Partial least-squares regression: a tutorial. Analytica chimica acta, 185:1-17, 1986.

[19] Mehmet Gönen and Ethem Alpaydın. Multiple kernel learning algorithms. The Journal of Machine Learning Research, 12:2211-2268, 2011.

[20] Richard P Good and S Joe Qin. On the stability of mimo ewma run-to-run controllers with metrology delay. IEEE Transactions on Semiconductor Manufacturing, 19(1):78-86, 2006.

[21] Graham C Goodwin and Kwai Sang Sin. Adaptive filtering prediction and control. Courier Dover Publications, 2013.

[22] Zhongsheng Hou and Shangtai Jin. Data-driven modelfree adaptive control for a class of mimo nonlinear discretetime systems. IEEE Transactions on Neural Networks, 22(12):2173-2188, 2011.

[23] S Joe Qin. Recursive pls algorithms for adaptive data modeling. Computers \& Chemical Engineering, 22(4):503$514,1998$.

[24] STEVEN A Juliano. Nonlinear curve fitting. Oxford University Press, 2001.

[25] Janusz Kacprzyk. Fuzzy regression analysis, volume 1. Physica-Verlag, 1992.

[26] Jyrki Kivinen, Alexander J Smola, and Robert C Williamson. Online learning with kernels. IEEE Transactions on Signal Processing, 52(8):2165-2176, 2004.

[27] Neil Lawrence, Matthias Seeger, Ralf Herbrich, et al. Fast sparse gaussian process methods: The informative vector machine. Advances in neural information processing systems, pages 625-632, 2003.

[28] Nan-Ying Liang, GuangBin Huang, Paramasivan Saratchandran, and Narasimhan Sundararajan. A fast and accurate online sequential learning algorithm for feedforward networks. IEEE Transactions on Neural Networks, 17(6):1411-1423, 2006.
[29] He Liu, Li Jia, Qing Liu, and Dao Huang. Batchto-batch control of batch processes based on multilayer recurrent fuzzy neural network. In Proceedings of the International Conference on Intelligent Systems and Knowledge Engineering. Atlantis Press, France, 2007.

[30] Yi Liu and Zengliang Gao. Real-time property prediction for an industrial rubber-mixing process with probabilistic ensemble gaussian process regression models. Journal of Applied Polymer Science, 132(6), 2015.

[31] Yi Liu, Zengliang Gao, Ping Li, and Haiqing Wang. Justin-time kernel learning with adaptive parameter selection for soft sensor modeling of batch processes. Industrial $\&$ Engineering Chemistry Research, 51(11):4313-4327, 2012.

[32] Yi Liu, Haiqing Wang, Jiang Yu, and Ping Li. Selective recursive kernel learning for online identification of nonlinear systems with NARX form. Journal of Process Control, 20(2):181 - 194, 2010.

[33] Zubin N Mevawalla, Gary S May, and Mark W Kiehlbauch. Neural network modeling for advanced process control using production data. IEEE Transactions on Semiconductor Manufacturing, 24(2):182-189, 2011.

[34] Laszlo Monostori and Janos Prohaszka. A step towards intelligent manufacturing: modelling and monitoring of manufacturing processes through artificial neural networks. CIRP Annals-Manufacturing Technology, 42(1):485-488, 1993.

[35] James Moyne, Enrique Del Castillo, and Arnon M Hurwitz. Run-to-run control in semiconductor manufacturing. CRC Press, 2010.

[36] S.R. Nassif. Modeling and forecasting of manufacturing variations. In Proceedings of the 5th International Workshop on Statistical Metrology, pages 2-10, 2000.

[37] Duy Nguyen-Tuong, Jan R Peters, and Matthias Seeger. Local gaussian process regression for real time online model learning. In Advances in Neural Information Processing Systems, pages 1193-1200, 2008.

[38] Duy Nguyen-Tuong, Matthias Seeger, and Jan Peters. Model learning with local gaussian process regression. Advanced Robotics, 23(15):2015-2034, 2009.

[39] Yun-Hsi O Chang and Bilal M Ayyub. Fuzzy regression methods-a comparative assessment. Fuzzy sets and systems, 119(2):187-203, 2001.

[40] Gianluigi Pillonetto, Alessandro Chiuso, and Giuseppe De Nicolao. Prediction error identification of linear systems: a nonparametric gaussian regression approach. Automatica, 47(2):291-305, 2011.

[41] Gianluigi Pillonetto and Giuseppe De Nicolao. A new kernelbased approach for linear system identification. Automatica, 46(1):81-93, 2010.

[42] Joaquin Quiñonero-Candela and Carl Edward Rasmussen. A unifying view of sparse approximate gaussian process regression. The Journal of Machine Learning Research, 6:1939-1959, 2005.

[43] Carl Edward Rasmussen. Gaussian processes in machine learning. In Advanced Lectures on Machine Learning, pages 63-71. Springer, 2004.

[44] Bernhard W Silverman et al. Some aspects of the spline smoothing approach to non-parametric regression curve fitting. Journal of the Royal Statistical Society, Series B, 47(1):1-52, 1985.

[45] X. Wang, Y. Liang, Q. Pan, and Y. Wang. Measurement random latency probability identification. IEEE Transactions on Automatic Control, PP(99):1-1, 2016. 
[46] Xiaoxu Wang, Yan Liang, Quan Pan, Chunhui Zhao, and Feng Yang. Design and implementation of gaussian filter for nonlinear system with randomly delayed measurements and correlated noises. Applied Mathematics and Computation, 232:1011 - 1024, 2014.

[47] Xiaoxu Wang, Bao Song, Yan Liang, and Quan Pan. Em-based adaptive divided difference filter for nonlinear system with multiplicative parameter. International Journal of Robust and Nonlinear Control, pages n/a-n/a, 2016. rnc.3674.

[48] Halbert White. Learning in artificial neural networks: A statistical perspective. Neural computation, 1(4):425-464, 1989.

[49] Yunfei Xu, Jongeun Choi, Sarat Dass, and Tapabrata Maiti. Efficient bayesian spatial prediction with mobile sensor networks using gaussian markov random fields. Automatica, 49(12):3520-3530, 2013.

\section{A Proof of Lemma 1}

PROOF. According to (1) and (4), we have for $0<l \leqslant$ $t$,

$$
\begin{aligned}
f_{t}(u) & =f_{0}(u)+\sum_{\tau=0}^{t-1} \omega_{\tau}, \\
z_{l} & =f_{0}(u)+\sum_{\tau=0}^{l-1} \omega_{\tau}+v_{l},
\end{aligned}
$$

which implies that for $f_{t}(u)$ and $Z_{t}(t>0)$ are jointly normally distributed. It is known that in this case the conditional mean is an estimate with the minimum MSE, thus the following result holds:

$$
\begin{aligned}
& \arg \min _{\hat{f}_{t}} \mathrm{E}\left[\left(\tilde{f}_{t}(u)\right)^{2}\right]=\mathrm{E}\left[f_{t}(u) \mid Z_{t}\right] \\
= & \mathbf{m}(u)+\operatorname{cov}\left(f_{t}(u), Z_{t}\right)\left[\operatorname{cov}\left(Z_{t}, Z_{t}\right)\right]^{-1}\left(Z_{t}-\mathrm{M}_{t}\right),
\end{aligned}
$$

where $\mathrm{M}_{t}$ is the mean vector as defined in (6). It is also straightforward to get

$$
\begin{aligned}
G_{t}(u)= & \operatorname{cov}\left(f_{t}(u), f_{t}(u)\right)-\operatorname{cov}\left(f_{t}(u), Z_{t}\right) \\
& \times\left[\operatorname{cov}\left(Z_{t}, Z_{t}\right)\right]^{-1}\left[\operatorname{cov}\left(f_{t}(u), Z_{t}\right)\right]^{\top} .
\end{aligned}
$$

From (5), we can get that for $0<l \leqslant t$,

$$
\begin{aligned}
\operatorname{cov}\left(f_{t}(u), f_{t}(u)\right) & =\sigma_{f}^{2}+\sigma_{\omega}^{2} t \\
\operatorname{cov}\left(f_{t}(u), z_{l}\right) & =\sigma_{f}^{2} \exp \left(-\frac{\left\|u-u_{l}\right\|^{2}}{h^{2}}\right)+\sigma_{\omega}^{2} l \\
\operatorname{cov}\left(z_{t}, z_{l}\right) & =\sigma_{f}^{2} \exp \left(-\frac{\left\|u_{t}-u_{l}\right\|^{2}}{h^{2}}\right)+\sigma_{\omega}^{2} l+\sigma_{v}^{2} \delta_{t l} .
\end{aligned}
$$

Thus, it can be obtained that

$$
\begin{aligned}
\operatorname{cov}\left(f_{t}(u), Z_{t}\right) & =\sigma_{f}^{2} K\left(u, U_{t}\right)+\sigma_{\omega}^{2} \boldsymbol{n}_{t}^{\mathrm{T}} \\
\operatorname{cov}\left(Z_{t}, Z_{t}\right) & =\sigma_{f}^{2} K\left(U_{t}, U_{t}\right)+\sigma_{\omega}^{2} \boldsymbol{N}_{t}+\sigma_{v}^{2} I_{t}
\end{aligned}
$$

where the related variables are defined in (6). Substituting the above results into (A.1) and (A.2) directly leads to $(6)$ and (7).

\section{B Proof of Theorem 1}

PROOF. First, define

$$
R_{t}(\boldsymbol{a}, \boldsymbol{b})= \begin{cases}\operatorname{cov}\left(f_{t}(\boldsymbol{a}), f_{t}(\boldsymbol{b}) \mid Z_{t}\right), & \text { if } t>0 \\ \operatorname{cov}\left(f_{t}(\boldsymbol{a}), f_{t}(\boldsymbol{b})\right), & \text { if } t=0\end{cases}
$$

It is easy to get $R_{0}(\boldsymbol{a}, \boldsymbol{b})=\sigma_{f}^{2} \exp \left(-\frac{\|\boldsymbol{a}-\boldsymbol{b}\|^{2}}{h^{2}}\right)$.

Letting $\hat{f}_{t}(u)=\mathrm{E}\left[f_{t}(u) \mid Z_{t}\right]$ which is an estimate of $f_{t}(u)$ with the minimum MSE, we have for $t>0$ that

$$
\begin{aligned}
& p\left(\left[f_{t}(\boldsymbol{a}), f_{t}(\boldsymbol{b}), f_{t}\left(u_{t+1}\right)\right]^{\mathrm{T}} \mid Z_{t}\right) \\
& \quad=\mathcal{N}\left(\left[\hat{f}_{t}(\boldsymbol{a}), \hat{f}_{t}(\boldsymbol{b}), \hat{f}_{t}\left(u_{t+1}\right)\right]^{\mathrm{T}}, A_{t}(\boldsymbol{a}, \boldsymbol{b})\right)
\end{aligned}
$$

where

$$
\begin{aligned}
& A_{t}(\boldsymbol{a}, \boldsymbol{b}) \\
& =\left[\begin{array}{ccc}
G_{t}(\boldsymbol{a}) & R_{t}(\boldsymbol{a}, \boldsymbol{b}) & R_{t}\left(\boldsymbol{a}, u_{t+1}\right) \\
R_{t}(\boldsymbol{b}, \boldsymbol{a}) & G_{t}(\boldsymbol{b}) & R_{t}\left(\boldsymbol{b}, u_{t+1}\right) \\
R_{t}\left(u_{t+1}, \boldsymbol{a}\right) & R_{t}\left(u_{t+1}, \boldsymbol{b}\right) & R_{t}\left(u_{t+1}, u_{t+1}\right)
\end{array}\right],
\end{aligned}
$$

and $p(\bullet)$ stands for the probability density distribution function. In addition, it holds that

$$
\begin{gathered}
p\left(\left[f_{t+1}(\boldsymbol{a}), f_{t+1}(\boldsymbol{b}), z_{t+1}\right]^{\mathrm{T}} \mid f_{t}(\boldsymbol{a}), f_{t}(\boldsymbol{b}), f_{t}\left(u_{t+1}\right)\right) \\
=\mathcal{N}\left(\left[f_{t}(\boldsymbol{a}), f_{t}(\boldsymbol{b}), f_{t}\left(u_{t+1}\right)\right]^{\mathrm{T}}, B(\boldsymbol{a}, \boldsymbol{b})\right)
\end{gathered}
$$

where

$$
B(\boldsymbol{a}, \boldsymbol{b})=\left[\begin{array}{ccc}
\sigma_{\omega}^{2} & \sigma_{\omega}^{2} & \sigma_{\omega}^{2} \\
\sigma_{\omega}^{2} & \sigma_{\omega}^{2} & \sigma_{\omega}^{2} \\
\sigma_{\omega}^{2} & \sigma_{\omega}^{2} & \sigma_{\omega}^{2}+\sigma_{v}^{2}
\end{array}\right]
$$


Thus, we have

$$
\begin{aligned}
& p\left(\left[f_{t+1}(\boldsymbol{a}), f_{t+1}(\boldsymbol{b}), z_{t+1}\right]^{\mathrm{T}} \mid Z_{t}\right) \\
= & \iiint p\left(\left[f_{t}(\boldsymbol{a}), f_{t}(\boldsymbol{b}), f_{t}\left(u_{t+1}\right)\right]^{\mathrm{T}} \mid Z_{t}\right) \\
& \times p\left(\left[f_{t+1}(\boldsymbol{a}), f_{t+1}(\boldsymbol{b}), z_{t+1}\right]^{\mathrm{T}} \mid f_{t}(\boldsymbol{a}), f_{t}(\boldsymbol{b}), f_{t}\left(u_{t+1}\right)\right) \\
& \times \mathrm{d}\left(f_{t}(\boldsymbol{a})\right) \mathrm{d}\left(f_{t}(\boldsymbol{b})\right) \mathrm{d}\left(f_{t}\left(u_{t+1}\right)\right) \\
= & \mathcal{N}\left(\left[\hat{f}_{t}(\boldsymbol{a}), \hat{f}_{t}(\boldsymbol{b}), \hat{f}_{t}\left(u_{t+1}\right)\right]^{\mathrm{T}}, A_{t}(\boldsymbol{a}, \boldsymbol{b})+B_{t}(\boldsymbol{a}, \boldsymbol{b})\right) .
\end{aligned}
$$

Then, it can be obtained that

$$
\begin{gathered}
p\left(\left[f_{t+1}(\boldsymbol{a}), f_{t+1}(\boldsymbol{b})\right]^{\mathrm{T}} \mid Z_{t+1}\right) \\
=\frac{p\left(\left[f_{t+1}(\boldsymbol{a}), f_{t+1}(\boldsymbol{b}), z_{t+1}\right]^{\mathrm{T}} \mid Z_{t}\right)}{p\left(z_{t+1} \mid Z_{t}\right)} \\
=\mathcal{N}\left(\left[\hat{f}_{t}(\boldsymbol{a})+\eta_{t}(\boldsymbol{a}), \hat{f}_{t}(\boldsymbol{b})+\eta_{t}(\boldsymbol{b})\right]^{\mathrm{T}}, C_{t}(\boldsymbol{a}, \boldsymbol{b})\right), \\
=\mathcal{N}\left(\left[\hat{f}_{t+1}(\boldsymbol{a}), \hat{f}_{t+1}(\boldsymbol{b})\right]^{\mathrm{T}}, C_{t}(\boldsymbol{a}, \boldsymbol{b})\right)
\end{gathered}
$$

where

$$
\eta_{t}(u)=\frac{R_{t}\left(u, u_{t+1}\right)}{R_{t}\left(u_{t+1}, u_{t+1}\right)+\sigma_{\omega}^{2}+\sigma_{v}^{2}}\left(z_{t+1}-\hat{f}_{t}\left(u_{t+1}\right)\right),
$$

and the $i j$-th entry of $C_{t}(\boldsymbol{a}, \boldsymbol{b})$, denoted by $\left\{C_{t}(\boldsymbol{a}, \boldsymbol{b})\right\}_{i j}$, is given as follows:

$$
\begin{aligned}
\left\{C_{t}(\boldsymbol{a}, \boldsymbol{b})\right\}_{11}= & R_{t}(\boldsymbol{a}, \boldsymbol{a})+\sigma_{\omega}^{2}-\frac{\left(R_{t}\left(\boldsymbol{a}, u_{t+1}\right)+\sigma_{\omega}^{2}\right)^{2}}{R_{t}\left(u_{t+1}, u_{t+1}\right)+\sigma_{\omega}^{2}+\sigma_{v}^{2}}, \\
\left\{C_{t}(\boldsymbol{a}, \boldsymbol{b})\right\}_{22}= & R_{t}(\boldsymbol{b}, \boldsymbol{b})+\sigma_{\omega}^{2}-\frac{\left(R_{t}\left(\boldsymbol{b}, u_{t+1}\right)+\sigma_{\omega}^{2}\right)^{2}}{R_{t}\left(u_{t+1}, u_{t+1}\right)+\sigma_{\omega}^{2}+\sigma_{v}^{2}}, \\
\left\{C_{t}(\boldsymbol{a}, \boldsymbol{b})\right\}_{12}= & \left\{C_{t}(\boldsymbol{a}, \boldsymbol{b})\right\}_{21}=R_{t+1}(\boldsymbol{a}, \boldsymbol{b}) \\
= & R_{t}(\boldsymbol{a}, \boldsymbol{b})+\sigma_{\omega}^{2} \\
& -\frac{\left(R_{t}\left(\boldsymbol{a}, u_{t+1}\right)+\sigma_{\omega}^{2}\right)\left(R_{t}\left(\boldsymbol{b}, u_{t+1}\right)+\sigma_{\omega}^{2}\right)}{R_{t}\left(u_{t+1}, u_{t+1}\right)+\sigma_{\omega}^{2}+\sigma_{v}^{2}} .
\end{aligned}
$$

From (B.1), we can directly get $\forall u \in \mathbb{U}$ that

$$
\begin{aligned}
\hat{f}_{t+1}(u) & =\hat{f}_{t}(u)+\eta_{t}(u) \\
R_{t+1}(u, u) & =R_{t}(u, u)+\sigma_{\omega}^{2}-\frac{\left(R_{t}\left(u, u_{t+1}\right)+\sigma_{\omega}^{2}\right)^{2}}{G_{t}\left(u_{t+1}\right)+\sigma_{\omega}^{2}+\sigma_{v}^{2}},
\end{aligned}
$$

where $R_{t}\left(u, u_{t+1}\right)$ can be calculated by the iteration in (B.2). Then, (11) holds according to the definition of $R_{t}(\boldsymbol{a}, \boldsymbol{b})$.

\section{Proof of Theorem 2}

PROOF. The existence can be shown if one solution that satisfies the requirement is found. Moreover, according to Lemma 2, we only need to show that $\operatorname{Tr}\left(G_{t}\left(U_{\mathrm{F}}\right)\right)$ is uniformly bounded. Consider the solution $\alpha_{t} \equiv 0$ for all $t>0$, which implies that $\hat{f}_{t}\left(U_{\mathrm{F}}\right)=\phi_{t}$ and $G_{t}\left(U_{\mathrm{F}}\right)=\Gamma_{t}$. Then, it follows that

$$
\begin{aligned}
& \mathrm{E}\left[\left\|\tilde{f}_{t}\left(U_{\mathrm{F}}\right)\right\|^{2}\right] \\
& =\operatorname{Tr}\left(G_{t}\left(U_{\mathrm{F}}\right)\right)=\operatorname{Tr}\left(\Gamma_{t}\right)=\mathrm{E}\left[\left\|f_{t}\left(U_{\mathrm{F}}\right)-\phi_{t}\right\|^{2}\right] \\
& =\sum_{i=1}^{\lambda} \mathrm{E}\left[\left\|f_{t}\left(\bar{u}_{i}\right)-\mathrm{E}\left[f_{t}\left(\bar{u}_{i}\right) \mid Z_{L, t}\right]\right\|^{2}\right] \\
& \leqslant \sum_{i=1}^{\lambda} \mathrm{E}\left[\left\|f_{t}\left(\bar{u}_{i}\right)-\mathrm{E}\left[f_{t}\left(\bar{u}_{i}\right) \mid z_{t}\right]\right\|^{2}\right] \\
& =\sum_{i=1}^{\lambda}\left(\sigma_{f}^{2}+\sigma_{\omega}^{2} t-\frac{\left(\sigma_{f}^{2} K\left(\bar{u}_{i}, u_{t}\right)+\sigma_{\omega}^{2} t\right)^{2}}{\sigma_{f}^{2}+\sigma_{\omega}^{2} t+\sigma_{v}^{2}}\right) \\
& \leqslant \sum_{i=1}^{\lambda} \frac{\left(\sigma_{f}^{2}+1\right)\left(\sigma_{v}^{2}+1\right)-\left(\sigma_{f}^{2} K\left(\bar{u}_{i}, u_{t}\right)+1\right)^{2}}{\sigma_{f}^{2}+\sigma_{v}^{2}} \\
& \leqslant \lambda\left(\frac{\sigma_{f}^{2} \sigma_{v}^{2}}{\sigma_{f}^{2}+\sigma_{v}^{2}}+1\right) \cdot
\end{aligned}
$$

This completes the proof.

\section{Proof of Theorem 3}

PROOF. From (15), it is straightforward to get

$$
\begin{aligned}
\tilde{f}_{t}(u)= & f_{t}(u)-\mathbf{m}(u)+W(u) \tilde{f}_{t}\left(U_{\mathrm{F}}\right) \\
& -W(u)\left(f_{t}\left(U_{\mathrm{F}}\right)-\mathbf{m}\left(U_{\mathrm{F}}\right)\right) \\
= & Q(u)+W(u) \tilde{f}_{t}\left(U_{\mathrm{F}}\right)+(1-W(u) \mathbf{1}) \sum_{\tau=0}^{t} \omega_{\tau}
\end{aligned}
$$

where

$$
\begin{aligned}
Q(u) & \triangleq f_{0}(u)-\mathbf{m}(u)-W(u)\left(f_{0}\left(U_{\mathrm{F}}\right)-\mathbf{m}\left(U_{\mathrm{F}}\right)\right) \\
& =\tilde{f}_{0}(u)-W(u) \tilde{f}_{0}\left(U_{\mathrm{F}}\right)
\end{aligned}
$$

Since $W(u) \mathbf{1}=1$, we have

$\mathrm{E}\left[\left\|\tilde{f}_{t}(u)\right\|^{2}\right] \leqslant 2 \mathrm{E}\left[\|Q(u)\|^{2}\right]+2\|W(u)\|^{2} \operatorname{Tr}\left(G_{t}\left(U_{\mathrm{F}}\right)\right)$, 
where

$\mathrm{E}\left[\|Q(u)\|^{2}\right]=\sigma_{f}^{2}\left(1-2 W(u) K\left(U_{\mathrm{F}}, u\right)+\lambda\|W(u)\|^{2}\right)$.

According to Theorem 2, $\operatorname{Tr}\left(G_{t}\left(U_{\mathrm{F}}\right)\right)$ is uniformly bounded. Thus, $\mathrm{E}\left[\left\|\tilde{f}_{t}(u)\right\|^{2}\right]$ must be uniformly bounded following (D.1). 